\title{
Immunohistochemical Expression and Prognostic Value of CD97 and Its Ligand CD55 in Primary Gallbladder Carcinoma
}

\author{
Jinsheng Wu, Liu Lei, Shaochuang Wang, Dianhua Gu, and Jianhuai Zhang \\ Department of Hepatobiliary Pancreatic Surgery, Huai'an First People's Hospital Nanjing Medical University, Jiangsu, \\ Huai'an 223300, China \\ Correspondence should be addressed to Jianhuai Zhang, jianhuai0517@sina.com
}

Received 25 November 2011; Accepted 25 January 2012

Academic Editor: Soldano Ferrone

Copyright (c) 2012 Jinsheng Wu et al. This is an open access article distributed under the Creative Commons Attribution License, which permits unrestricted use, distribution, and reproduction in any medium, provided the original work is properly cited.

Background. CD97 as a member of the EGF-TM7 family with adhesive properties plays an important role in tumor aggressiveness by binding its cellular ligand CD55, which is a complement regulatory protein expressed by cells to protect them from bystander complement attack. Previous studies have shown that CD97 and CD55 both play important roles in tumor dedifferentiation, migration, invasiveness, and metastasis. The aim of this study was to investigate CD97 and CD55 expression in primary gallbladder carcinoma (GBC) and their prognostic significance. Methods. Immunohistochemistry was used to investigate the expression of CD97 and CD55 proteins in 138 patients with GBC. Results. CD97 and CD55 were absent or only weakly expressed in the normal epithelium of the gallbladder but in $69.6 \%(96 / 138)$ and $65.2 \%(90 / 138)$ of GBC, respectively, remarkably at the invasive front of the tumors. In addition, CD97 and CD55 expressions were both significantly associated with high histologic grade (both $P=$ $0.009)$, advanced pathologic T stage $(P=0.01$ and 0.009 , resp.) and clinical stage (both $P=0.009)$, and positive venous/lymphatic invasion (both $P=0.009$ ). Multivariate analyses showed that CD97 (hazard ratio, 3.236; $P=0.02$ ) and CD55 (hazard ratio, 3.209; $P=0.02$ ) expressions and clinical stage (hazard ratio, 3.918; $P=0.01$ ) were independent risk factor for overall survival. Conclusion. Our results provide convincing evidence for the first time that the expressions of CD97 and CD55 are both upregulated in human GBC. The expression levels of CD97 and CD55 in GBC were associated with the severity of the tumor. Furthermore, CD97 and CD55 expressions were independent poor prognostic factors for overall survival in patients with GBC.

\section{Introduction}

Primary gallbladder carcinoma (GBC) is the sixth most common gastrointestinal cancer in Asian and Western countries [1]. This carcinoma has extremely poor prognosis and increasing incidence worldwide because of its inherent biology and often advanced stage at diagnosis, despite the recent advances in diagnostic modalities. GBC has a great propensity to directly invade the liver, and it also frequently metastasizes to the liver and pericholedochal lymph nodes [2]. There are no adjuvant chemotherapeutic combinations widely accepted for GBC due to their toxicity, drug resistance, and limited efficacy. Curative surgical approaches are still the principal treatment, but the prognosis of GBC remains poor with respect to postsurgical 5-year survival rates [3]. Thus, the search for new prognostic markers for GBC patients is important to allow the assessment of metastasis and to provide the opportunity for adequate postoperative treatment in high-risk patients.

CD97 is a member of the epidermal-growth-factor (EGF-) seven-span transmembrane (TM7) family with adhesive properties [4]. This family includes EMR1, EMR2, EMR3, ETL, and the mouse microglia marker F4/80, which are characterized by an extended extracellular region with a variable number of EGF domains. So far, CD97 is the only EGF-TM7 family member of which a ligand has been identified [5]. Via its EGF domain region, CD97 binds CD55 (also named as decay accelerating factor, DAF), a glycosylphosphatidylinositol-anchored protein, which regulates complement activation pathway [6]. As in the case of many glycosylphosphatidylinositol-anchored proteins, CD55 is either bound to the cell membrane or released from the membrane into the microenvironment. CD55 binds to $\mathrm{C} 3$ convertases generated from both the classic and 
alternative complement pathways, prevents $\mathrm{C} 3 \mathrm{~b}$ deposition, thus inhibiting the formation of membrane attack complex. Physiologically CD55 serves as an inhibitor of the complement system [7]. Various evidences showed that CD97 and CD55 both play important roles in tumor dedifferentiation, migration, invasiveness, and metastasis [8-12]. For example, the expressions of CD97 and CD55 in rectal tumor tissues were significantly higher than those in normal colorectal tissues. The patients with recurrence and/or metastasis had significantly higher expressions of CD97 in tumor cells and CD55 in stroma at the invasion front than those patients without recurrence and/or metastasis [8]. Durrant et al. also demonstrated that the colorectal cancer patients whose tumors overexpress CD55 have a significantly reduced 7-year survival [10]. The findings of Liu et al. suggest that CD97 may play a role in the development and invasion of gastric carcinomas by binding its cellular ligand CD55. Detection of the CD97 expression in the tumor margin could be referred to as the molecular edge of gastric carcinomas. They also suggested that CD97 and CD55 could be useful as molecular markers for prognosis and therapy of gastric carcinoma patients $[11,12]$. However, the expression and clinical impact of CD97 and its ligand CD55 in GBC have not been previously investigated. Herein, we found that the expressions of CD97 and CD55 are both upregulated in human GBC. Their expression levels in GBC were both associated with the severity of the tumor. Furthermore, CD97 and CD55 expressions were independent poor prognostic factors for overall survival in patients with GBC.

\section{Materials and Methods}

2.1. Patients and Tissue Samples. This study was approved by the Research Ethics Committee of the Huai'an First People's Hospital Affiliated to Nanjin Medical University, Huai'an, Jiangsu, China. Written informed consent was obtained from all of the patients. All specimens were handled and made anonymous according to the ethical and legal standards.

We retrospectively investigated 138 patients (60 men and 78 women) with GBC who underwent surgical resection between March 1999 and January 2010 at Department of Hepatobiliary Pancreatic Surgery, the Huai'an First People's Hospital Affiliated to Nanjin Medical University, Huai'an, Jiangsu, China. No preoperative chemotherapy or radiotherapy had been performed in any of these cases. Patients, who died of diseases not directly related to their GBC or due to unexpected events, were excluded from this study. The mean age of the patients was 68 years (range, 3688 years). All tumors were diagnosed as adenocarcinoma and defined as primary tumor arising from the gallbladder. Tumor stage was classified according to the American Joint Committee on Cancer system. All protocols were approved by the institutional review board. The GBC specimens were examined by routine hematoxylin and eosin staining. The specimens were graded into well (G1), moderately (G2), poorly differentiated (G3), and undifferentiated (G4) adenocarcinoma according to the World Health Organization classification.
2.2. Immunohistochemistry Assay. Formalin-fixed, paraffinembedded, sectioned tissues ( $4 \mu \mathrm{m}$ thick) were immunostained using the Labelled Streptavidin Biotin 2 System (BioGenex; San Ramon, CA, USA). Following peroxidase blocking with $0.3 \% \mathrm{H}_{2} \mathrm{O}_{2}$ /methanol for $30 \mathrm{~min}$, specimens were blocked with phosphate-buffered saline (PBS) containing 5\% normal horse serum (Vector Laboratories Inc., Burlingame, CA, USA). All specimens were incubated with monoclonal antibodies against CD55 (clone 143-30, BD Biosciences, Heidelberg, Germany) and CD97 (MEM180, Biermann, Germany) at dilutions of $1: 100$, respectively, overnight at $4{ }^{\circ} \mathrm{C}$. Then the specimens were briefly washed in PBS and incubated at room temperature with the anti-mouse antibody and avidin-biotin peroxidase (Vector Laboratories Inc., Burlingame, CA, USA). The specimens were then washed in PBS and color-developed by diaminobenzidine solution (Dako Corporation, Carpinteria, CA, USA). After washing with water, specimens were counterstained with Meyer's hematoxylin (Sigma Chemical Co., St. Louis, MO, USA). Nonimmune IgG was used as negative control antibody for immunohistochemical staining.

Assessment of immunohistochemical staining was evaluated by two independent pathologists. The numbers of CD97-positive and CD55-positive cells in 10 representative microscopic fields were counted. The percentage of positive cells was judged followed by Remmele W's criteria [13] ranging from 1 to $4(1,<10 \% ; 2,10$ to $50 \% ; 3,51$ to $80 \%$; $>80 \%$ positive tumor cells), and the level of staining intensity was estimated to grades between 0 and 3 ( 0 , negative; 1 , weak; 2 , moderate; 3 , strong staining). The product of positive cells and staining intensity gave the score (0 to 12$)$. We considered the samples positive if the score was higher than 4 .

2.3. Statistical Analysis. All computations were carried out using the software of SPSS version13.0 for Windows (SPSS Inc., IL, USA). Categorical data were examined by use of the chi-squared test. Overall survival (OS) was defined from the date of surgery to the date of death by GBC. Survival curves were plotted using the Kaplan-Meier method and analyzed using the log-rank test. Cox proportional hazards model was performed to identify prognostic factors for survival. Differences were considered statistically significant when $P$ was less than 0.05 .

\section{Results}

3.1. Immunohistochemical Staining of CD97 and CD55 Proteins in GBC Tissues. Our analysis determined that CD97 and CD55 were absent or only weakly expressed in the normal epithelium of the gallbladder (Figures 1(A) and $1(B)$, resp.) but in $69.6 \%(96 / 138)$ and $65.2 \%(90 / 138)$ of $\mathrm{GBC}$, respectively, remarkably at the invasive front of the tumors. The expression pattern of CD97 was significantly correlated with that of CD55 $(r=0.86, P=0.002)$. CD97 immunoreactivity was observed in the cytoplasm of tumor cells (Figures 1(C) and 1(D)), and CD55 immunoreactivity was observed on the cell membrane of tumor cells (Figures $1(\mathrm{E})$ and $1(\mathrm{~F})$ ), both with varying levels of percentage tumor staining and intensities. 

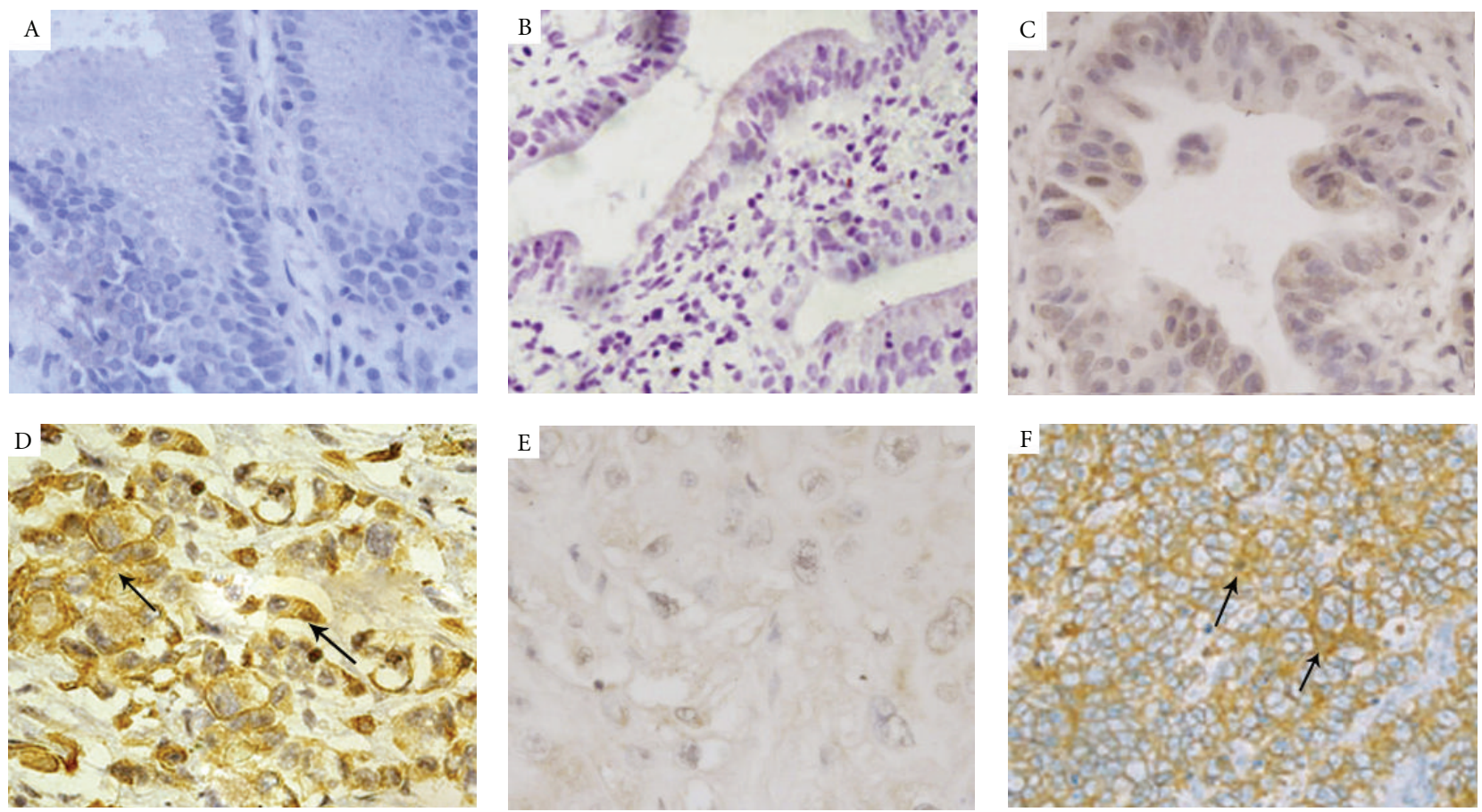

FIGURE 1: Immunohistochemical stainings for CD97 and CD55 in GBC tissues (original magnification $\times 200$ ). (A) and (B) CD97 and CD55 stainings were negative in nontumor brain tissues; (C) weak CD97 positive expression was found in the cytoplasm at various levels in GBC tissues with low clinical stage; (D) strong CD97 positive expression was found in the cytoplasm at various levels in GBC tissues with high clinical stage; (E) weak CD55 positive expression was found in the cell membrane at various levels in GBC tissues with low clinical stage; (F) strong CD55 positive expression was found in the cell membrane at various levels in GBC tissues with high clinical stage.

3.2. Correlation of CD97 and CD55 Expression with Clinicopathologic Factors. We next analyze the correlation between CD97 and CD55 expression and various clinicopathologic factors that can affect the prognosis of patients with GBC. The results are summarized in Table 1 . There was no association of the expression patterns of CD97 and CD55 with age and sex. Interestingly, the expression of CD97 and CD55 in moderately or poorly differentiated GBC was significantly higher than that in well-differentiated GBC (both $P=0.009$ ), indicating a correlation between their expressions and aggressive histologic grade of GBC. In addition, expression of CD97 and CD55 was also significantly associated with advanced pathologic $\mathrm{T}$ stage $(P=$ 0.01 and 0.009 , resp.), advanced clinical stage (both $P=$ 0.009 ), and positive venous/lymphatic invasion (both $P=$ 0.009). Other clinicopathologic factors such as lymph node metastasis, distant metastasis, and perineural invasion were not significantly related to CD97 and CD55 expression.

\subsection{Correlation of CD97 and CD55 Expression with Survival.} To further investigate the clinical usefulness of CD97 and CD55 expression in GBC, we compared OS according to various clinicopathologic factors including expression levels of CD97 and CD55. Age and sex did not influence the OS. However, patients with high histologic grade, advanced pathologic $\mathrm{T}$ stage, clinical stage, nodal metastasis, positive venous/lymphatic invasion, CD97, and CD55 expression were significantly correlated with short survival. The median survival time of patients with GBC was 38 months (range,
1-119 months). The survival curves according to CD97 and CD55 expression are shown in Figures 2(a) and 2(b), respectively. The OS rates of CD97 negative and CD97 positive were $42.9 \%$ and $12.5 \%$, respectively (Figure $2(\mathrm{a})$ ) $(P=0.02)$, and the OS rates of CD55 negative and CD55 positive were $45.8 \%$ and $17.8 \%$, respectively (Figure 2(b)) $(P=0.02)$. These analyses with Kaplan-Meier method clearly showed the significant impact of CD97 and CD55 expression on clinical outcome.

To estimate the clinical significance of various prognostic factors that might influence survival, univariate analyses were performed. As summarized in Table 2, advanced pathologic T stage $(P=0.008)$, advanced clinical stage $(P=$ $0.001)$, nodal metastasis $(P<0.001)$, distant metastasis $(P=$ $0.001)$, positive venous/lymphatic invasion $(P=0.001)$, positive perineural invasion $(P=0.01)$, positive CD97 $(P=$ $0.005)$ and CD55 expression $(P=0.007)$, and the coexpression of CD97 and CD55 $(P=0.008)$ were statistically significant risk factors affecting OS of patients with GBC. To determine the independent prognostic impacts of these various factors, multivariate analyses were performed using the Cox proportional hazards model. Multivariate analyses showed that CD97 (hazard ratio, 3.236; $P=0.02$ ) and CD55 (hazard ratio, 3.209; $P=0.02$ ) expressions, the coexpression of CD97 and CD55 $(P=0.01)$, and clinical stage (hazard ratio, 3.918; $P=0.01$ ) were independent risk factors for overall survival (Table 3). These results indicate that CD97 and CD55 expression might be potential histopathological biomarkers to predict patient survival. 
TABLE 1: Correlation of CD97 and CD55 expressions with conventional clinicopathological parameters in 138 patients with GBC.

\begin{tabular}{|c|c|c|c|c|c|c|c|}
\hline \multirow{2}{*}{ Factor } & \multirow{2}{*}{ Number } & \multicolumn{2}{|c|}{ CD97 expression $(n, \%)$} & \multirow{2}{*}{$P$} & \multicolumn{2}{|c|}{ CD55 expression $(n, \%)$} & \multirow{2}{*}{$P$} \\
\hline & & Negative & Positive & & Negative & Positive & \\
\hline \multicolumn{8}{|l|}{ Gender } \\
\hline Male & 60 & $16(26.7)$ & $44(73.3)$ & \multirow{2}{*}{ NS } & $20(33.3)$ & $40(66.7)$ & \multirow{2}{*}{ NS } \\
\hline Female & 78 & $26(33.3)$ & $52(66.7)$ & & $28(35.9)$ & $50(64.1)$ & \\
\hline Age at diagnosis & & $67.2 \pm 1.3$ & $69.7 \pm 1.8$ & NS & $67.0 \pm 1.5$ & $69.8 \pm 1.9$ & NS \\
\hline \multicolumn{8}{|c|}{ Histological grade } \\
\hline G1 & 18 & $18(100.0)$ & $0(0.0)$ & \multirow{4}{*}{0.009} & $18(100.0)$ & $0(0.0)$ & \multirow{4}{*}{0.009} \\
\hline G2 & 84 & $23(27.4)$ & $61(72.6)$ & & $26(31.0)$ & $58(69.0)$ & \\
\hline G3 & 34 & $1(2.9)$ & $33(97.1)$ & & $4(11.8)$ & $30(88.2)$ & \\
\hline G4 & 2 & $0(0.0)$ & $2(100.0)$ & & $0(0.0)$ & $2(100.0)$ & \\
\hline \multicolumn{8}{|l|}{ Pathologic T stage } \\
\hline $\mathrm{T} 1$ & 32 & $23(71.9)$ & $9(29.1)$ & \multirow{4}{*}{0.01} & $25(78.1)$ & $7(21.9)$ & \multirow{4}{*}{0.009} \\
\hline $\mathrm{T} 2$ & 64 & $13(20.3)$ & $51(79.7)$ & & $17(26.6)$ & $47(73.4)$ & \\
\hline T3 & 36 & $6(16.7)$ & $30(83.3)$ & & $6(16.7)$ & $30(83.3)$ & \\
\hline $\mathrm{T} 4$ & 6 & $0(0.0)$ & $6(100.0)$ & & $0(0.0)$ & $6(100.0)$ & \\
\hline \multicolumn{8}{|l|}{ Clinical stage } \\
\hline $\mathrm{I}$ & 60 & $30(50.0)$ & $30(50.0)$ & \multirow{4}{*}{0.009} & $35(58.3)$ & $25(41.7)$ & \multirow{4}{*}{0.009} \\
\hline II & 48 & $10(20.8)$ & $38(79.2)$ & & $11(22.9)$ & $37(77.1)$ & \\
\hline III & 18 & $2(11.1)$ & $16(88.9)$ & & $2(11.1)$ & $16(88.9)$ & \\
\hline IV & 12 & $0(0.0)$ & $12(100.0)$ & & $0(0.0)$ & $12(100.0)$ & \\
\hline \multicolumn{8}{|l|}{ Nodal metastasis } \\
\hline Negative & 102 & $31(30.4)$ & $71(69.6)$ & \multirow{2}{*}{ NS } & $35(34.3)$ & $67(65.7)$ & \multirow{2}{*}{ NS } \\
\hline Positive & 36 & $11(30.6)$ & $25(69.4)$ & & $13(36.1)$ & $23(63.9)$ & \\
\hline \multicolumn{8}{|l|}{ Distant metastasis } \\
\hline Negative & 126 & $38(30.2)$ & $88(69.8)$ & \multirow{2}{*}{ NS } & $43(34.1)$ & $83(65.9)$ & \multirow{2}{*}{ NS } \\
\hline Positive & 12 & $4(33.3)$ & $8(66.7)$ & & $5(41.7)$ & $7(58.3)$ & \\
\hline \multicolumn{8}{|c|}{$\begin{array}{l}\text { Venous/lymphatic } \\
\text { invasion }\end{array}$} \\
\hline Negative & 48 & $28(58.3)$ & $20(41.7)$ & \multirow{2}{*}{0.009} & $28(58.3)$ & $20(41.7)$ & \multirow{2}{*}{0.009} \\
\hline Positive & 90 & $14(15.6)$ & $76(84.4)$ & & $20(22.2)$ & $70(77.8)$ & \\
\hline \multicolumn{8}{|c|}{ Perineural invasion } \\
\hline Negative & 66 & $26(39.4)$ & $40(60.6)$ & \multirow{2}{*}{ NS } & $22(33.3)$ & $44(66.7)$ & NS \\
\hline Positive & 72 & $24(33.3)$ & $48(66.7)$ & & $26(36.1)$ & $46(63.9)$ & \\
\hline
\end{tabular}

TABLE 2: Univariate analysis of the associations of prognosis with various clinicopathologic parameters in patients with PGC.

\begin{tabular}{lccc}
\hline Features & & OS & 95\% CI \\
\hline Gender & HR & $0.312-1.326$ & 0.3 \\
Age at diagnosis & 0.696 & $0.383-1.508$ & 0.2 \\
Histological grade & 33.291 & $0.869-1767.639$ & 0.06 \\
Pathologic T stage & 2.923 & $1.385-8.763$ & 0.008 \\
Clinical stage & 4.951 & $2.015-10.278$ & 0.001 \\
Nodal metastasis & 3.009 & $1.172-8.638$ & $<0.001$ \\
Distant metastasis & 8.093 & $2.168-22.679$ & 0.001 \\
Venous/lymphatic invasion & 11.662 & $2.908-51.338$ & 0.001 \\
Perineural invasion & 3.921 & $1.087-8.692$ & 0.01 \\
CD97 expression & 3.039 & $1.122-7.165$ & 0.005 \\
CD55 expression & 2.763 & $0.928-6.927$ & 0.007 \\
CD97 positive/CD55 positive & 2.591 & $0.882-6.626$ & 0.008 \\
\hline
\end{tabular}




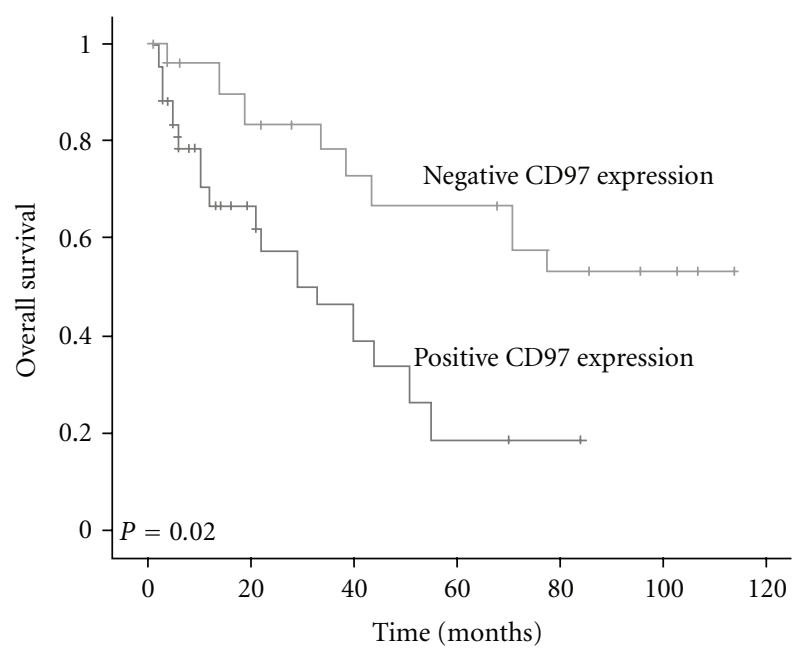

(a)

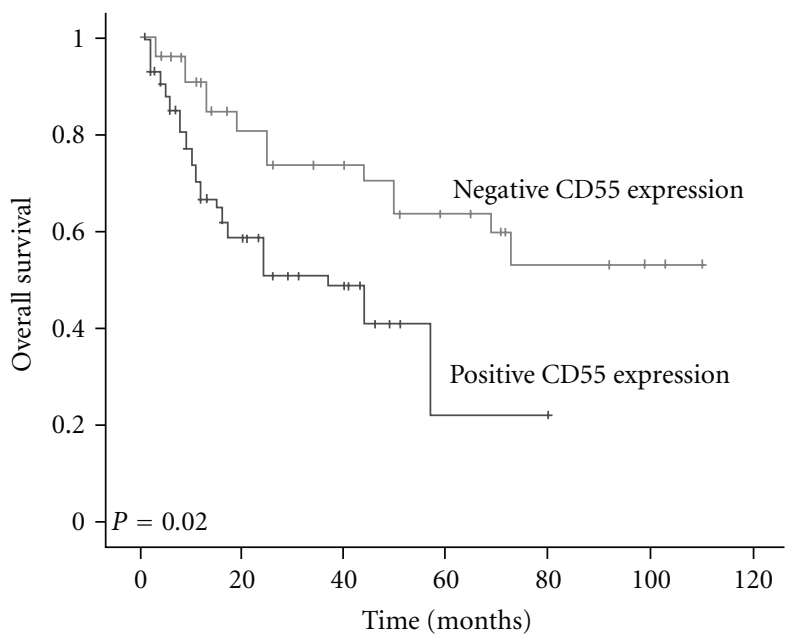

(b)

Figure 2: Correlation of CD97 (a) and CD55 (b) expression with the overall survival rates in 138 patients with GBC (compared with log-rank test).

TABLE 3: Multivariate analysis of the associations of prognosis with various clinicopathologic parameters in patients with PGC.

\begin{tabular}{lccc}
\hline Features & \multicolumn{3}{c}{ OS } \\
& HR & $95 \%$ CI & $P$ \\
\hline Pathologic T stage & 0.202 & $0.007-5.109$ & 0.4 \\
Clinical stage & 3.918 & $0.666-8.608$ & 0.01 \\
Nodal metastasis & 1.058 & $0.532-3.596$ & 0.7 \\
Distant metastasis & 1.926 & $0.512-4.053$ & 0.6 \\
Venous/lymphatic & 1.851 & $0.512-4.106$ & 0.6 \\
invasion & 0.662 & $0.228-1.986$ & 0.4 \\
Perineural invasion & 3.236 & $1.098-8.651$ & 0.02 \\
CD97 expression & 3.209 & $0.993-8.625$ & 0.02 \\
CD55 expression & 4.027 & $0.696-8.881$ & 0.01 \\
CD97 positive/CD55 & &
\end{tabular}

\section{Discussion}

GBC is one of the most aggressive malignancies. It is usually diagnosed at an advanced stage, and the prognosis remains poor despite the improvement in imaging techniques and aggressive surgical treatment. Therefore, identification of biologic markers associated with advanced stages of tumor progression is necessary for early diagnosis and the discovery of a therapeutic target. To our knowledge, the ideal candidate tumor markers must satisfy two criteria. First, it should be selectively expressed solely on tumor cells, not surrounding normal cells. Second, it should be specifically present and/or overexpressed on various cancer tissues. There are three main findings in the present study. At first, strong CD97 and CD55 stainings were both observed at the invasive tumor front but absent or weak in the normal epithelium of the gallbladder. Then, CD97 and CD55 expression in GBC was significantly correlated with tumor progression and clinicopathologic features. Immunohistochemical analysis of a large set of GBCs revealed that $69.6 \%$ and $65.2 \%$ of GBCs were immunopositive for CD97 and CD55, respectively. Their expressions in moderately or poorly differentiated GBC were significantly higher than that in well-differentiated GBC. In addition, expression of CD97 and CD55 was also significantly associated with advanced pathologic $\mathrm{T}$ stage, advanced clinical stage, and positive venous/lymphatic invasion, suggesting that their expression might be of clinical relevance in the aggressiveness of GBC. Thirdly, the impact of CD97 and CD55 expression on clinical outcome was assessed by Kaplan-Meier analyses. OS rates of CD97 negative and CD55 negative were both significantly higher than those of CD97 positive and CD55 positive. Univariate and multivariate analyses clearly demonstrated that CD97 and CD55 expressions were statistically significant risk factors affecting OS of patients with GBC and were independent risk factors predicting short OS. These findings suggest that CD97 and CD55 are ideal candidate tumor markers for GBC.

CD97 is a member of the EGF-7TM subfamily of class B G-protein-coupled receptors. It consists of a variable number of N-terminally located EGF domains, a long extracellular stalk, the 7-transmembrane domain, and a short intracellular C-terminal region [14-16]. It has been demonstrated that CD97 participates in the migration and invasion of tumor cells, which can be strengthened by CD55, the ligand for CD97 [17]. In gastric tumor tissues, the expression of CD55 was well correlated with that of CD97. Particularly, strong staining of CD97 and CD55 was observed in the cytoplasm of the signet ring cell carcinoma of patients, which suggests that CD97 may be involved in the remodelling of adhesive contacts of the invading tumor cells by direct receptor-ligand interactions [18]. In colorectal carcinomas, CD97 and CD55 are overexpressed in the tumor environment. At the tumorstroma interface, CD97 is preferentially overexpressed in tumor cells. By direct receptor-ligand interactions, CD97 may be involved in the remodeling of adhesive contacts 
of the invading tumor cells with the extracellular matrix. In vitro colorectal tumor cell lines expressing CD55 and endothelial cells deposit CD55 into the extracellular matrix. Perhaps such exogenic signals from the specific tumor environment regulate the cellular distribution of CD97 and consequently activation of its unknown target genes [19]. In medullary thyroid carcinomas, CD97 transcriptional activity was high in advanced stages of tumor tissues such as pT3/4. The pT1/2 tumors with exclusive intrathyroidal growth revealed weak CD97 expression. CD55 gene expression was significantly lower in normal C-cells than in tumor tissues, and all medullary thyroid carcinomas displayed strong and specific CD55 immunostaining [20]. In agreement with these previous reports on other tumors, our study demonstrated an overexpression of CD97 and CD55 in human GBC tissues, which also correlated significantly to the aggressive disease features including high histologic grade, pathologic T stage, clinical stage, positive venous/lymphatic invasion, and poor postoperative prognosis. These findings suggest that the two proteins may play an important role in tumor growth and progression of GBC. When it comes to the relevance of CD97 and CD55 to tumor treatment, Zhao et al. [21] reported that CD55 may augment the therapeutic effect of herceptin against lung carcinoma cells; Geis et al. [22] indicated that the inhibition of CD55 expression by siRNA could sensitize tumor cells to complement attack in vitro; Dzietczenia et al. [23] demonstrated that the CD55 expression in patients with CD20+ non-Hodgkin's lymphoma could be clinically helpful to predict the response to rituximab therapy. The involvement of CD97 and CD55 expression in the therapy of GBC patients needs to be further investigated.

In conclusion, this preliminary study has demonstrated for the first time that CD97 and CD55 proteins might be potential histopathological biomarkers for GBC. Their expression levels are associated with the severity of GBC. Furthermore, CD97 and CD55 expressions were independent poor prognostic factors for overall survival in patients with GBC.

\section{Authors' Contribution}

J. Wu and L. Lei equally contributed to this study.

\section{References}

[1] W. S. Kim, K. T. Jang, D. W. Choi et al., "Clinicopathologic analysis of adenosquamous/squamous cell carcinoma of the gallbladder," Journal of Surgical Oncology, vol. 103, no. 3, pp. 239-242, 2011.

[2] F. Miura, T. Asano, H. Amano et al., "New prognostic factor influencing long-term survival of patients with advanced gallbladder carcinoma," Surgery, vol. 148, no. 2, pp. 271-277, 2010.

[3] Z. H. Hu, Z. W. Li, L. Shen, M. Zhang, and S. S. Zheng, "Surgical therapy and prognosis of sarcomatoid carcinoma of the gallbladder," Hepatobiliary and Pancreatic Diseases International, vol. 9, no. 2, pp. 175-179, 2010.

[4] M. J. Kwakkenbos, E. N. Kop, M. Stacey et al., "The EGF-TM7 family: a postgenomic view," Immunogenetics, vol. 55, no. 10, pp. 655-666, 2004.
[5] S. Lea, "Interactions of CD55 with non-complement ligands," Biochemical Society Transactions, vol. 30, no. 6, pp. 1014-1019, 2002.

[6] L. Visser, A. F. De Vos, J. Hamann et al., "Expression of the EGF-TM7 receptor CD97 and its ligand CD55 (DAF) in multiple sclerosis," Journal of Neuroimmunology, vol. 132, no. 1-2, pp. 156-163, 2002.

[7] R. M. Hoek, D. De Launay, E. N. Kop et al., "Deletion of either CD55 or CD97 ameliorates arthritis in mouse models," Arthritis and Rheumatism, vol. 62, no. 4, pp. 1036-1042, 2010.

[8] S. L. Han, C. Xu, X. L. Wu, J. L. Li, Z. Liu, and Q. Q. Zeng, "The impact of expressions of CD97 and its ligand CD55 at the invasion front on prognosis of rectal adenocarcinoma," International Journal of Colorectal Disease, vol. 25, no. 6, pp. 695-702, 2010.

[9] R. D. Loberg, K. J. Wojno, L. L. Day, and K. J. Pienta, "Analysis of membrane-bound complement regulatory proteins in prostate cancer," Urology, vol. 66, no. 6, pp. 1321-1326, 2005.

[10] L. G. Durrant, M. A. Chapman, D. J. Buckley, I. Spendlove, R. A. Robins, and N. C. Armitage, "Enhanced expression of the complement regulatory protein CD55 predicts a poor prognosis in colorectal cancer patients," Cancer Immunology, Immunotherapy, vol. 52, no. 10, pp. 638-642, 2003.

[11] Y. Liu, L. Chen, S. Peng et al., "The expression of CD97EGF and its ligand CD55 on marginal epithelium is related to higher stage and depth of tumor invasion of gastric carcinomas," Oncology reports., vol. 14, no. 6, pp. 1413-1420, 2005.

[12] Y. Liu, L. Chen, S. Y. Peng, Z. X. Chen, and C. Hoang-Vu, "Role of CD97stalk and CD55 as molecular markers for prognosis and therapy of gastric carcinoma patients," Journal of Zhejiang University: Science, vol. 6, no. 9, pp. 913-918, 2005.

[13] W. Remmele and H. E. Stegner, "Recommendation for uniform definition of an immunoreactive score (IRS) for immunohistochemical estrogen receptor detection (ER-ICA) in breast cancer tissue," Pathologe, vol. 8, no. 3, pp. 138-140, 1987.

[14] W. S. Kim, D. W. Choi, D. D. You, C. Y. Ho, J. S. Heo, and S. H. Choi, "Risk factors influencing recurrence, patterns of recurrence, and the efficacy of adjuvant therapy after radical resection for gallbladder carcinoma," Journal of Gastrointestinal Surgery, vol. 14, no. 4, pp. 679-687, 2010.

[15] T. Mustafa, A. Eckert, T. Klonisch et al., "Expression of the epidermal growth factor seven-transmembrane member CD97 correlates with grading and staging in human oral squamous cell carcinomas," Cancer Epidemiology Biomarkers and Prevention, vol. 14, no. 1, pp. 108-119, 2005.

[16] M. J. Kwakkenbos, W. Pouwels, M. Matmati et al., "Expression of the largest CD97 and EMR2 isoforms on leukocytes facilitates a specific interaction with chondroitin sulfate on $\mathrm{B}$ cells," Journal of Leukocyte Biology, vol. 77, no. 1, pp. 112-119, 2005.

[17] M. Wobus, B. Vogel, E. Schmücking, J. Hamann, and G. Aust, "N-glycosylation of CD97 within the EGF domains is crucial for epitope accessibility in normal and malignant cells as well as CD55 ligand binding," International Journal of Cancer, vol. 112, no. 5, pp. 815-822, 2004.

[18] D. Liu, B. Trojanowicz, Y. Radestock et al., "Role of CD97 isoforms in gastric carcinoma," International Journal of Oncology, vol. 36, no. 6, pp. 1401-1408, 2010.

[19] M. Wobus, O. Huber, J. Hamann, and G. Aust, "CD97 overexpression in tumor cells at the invasion front in colorectal cancer (CC) is independently regulated of the canonical Wnt pathway," Molecular Carcinogenesis, vol. 45, no. 11, pp. 881886, 2006. 
[20] T. Mustafa, T. Klonisch, S. Hombach-Klonisch et al., "Expression of CD97 and CD55 in human medullary thyroid carcinomas," International journal of oncology, vol. 24, no. 2, pp. 285-294, 2004.

[21] W. P. Zhao, B. Zhu, Y. Z. Duan, and Z. T. Chen, "Neutralization of complement regulatory proteins CD55 and CD59 augments therapeutic effect of herceptin against lung carcinoma cells," Oncology Reports, vol. 21, no. 6, pp. 1405$1411,2009$.

[22] N. Geis, S. Zell, R. Rutz et al., "Inhibition of membrane complement inhibitor expression (CD46, CD55, CD59) by siRNA sensitizes tumor cells to complement attack in vitro," Current Cancer Drug Targets, vol. 10, no. 8, pp. 922-931, 2010.

[23] J. Dzietczenia, T. Wróbel, G. Mazur, R. Poręba, B. Jaźwiec, and K. Kuliczkowski, "Expression of complement regulatory proteins: CD46, CD55, and CD59 and response to rituximab in patients with CD20(+) non-Hodgkin's lymphoma," Medical Oncology, vol. 27, no. 3, pp. 743-746, 2010. 


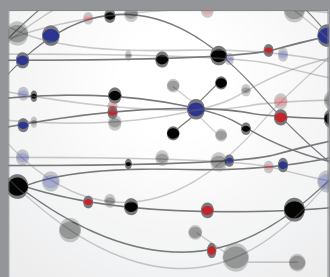

The Scientific World Journal
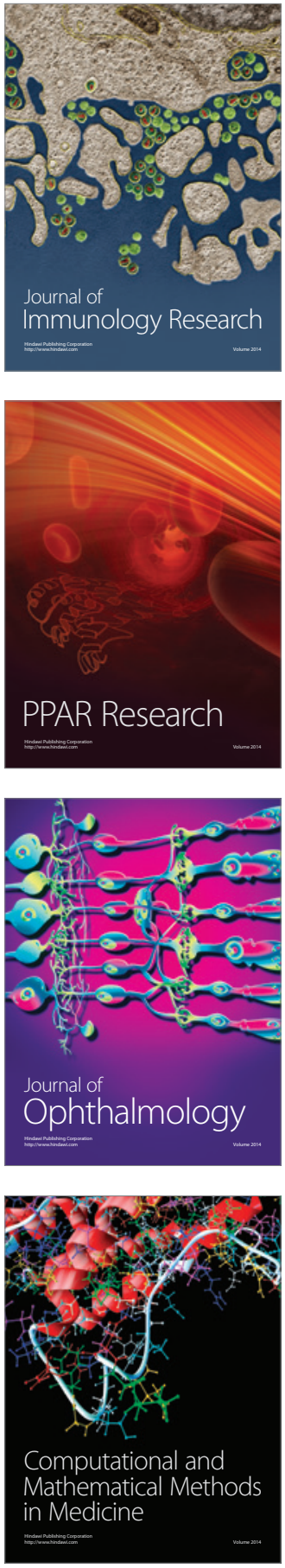

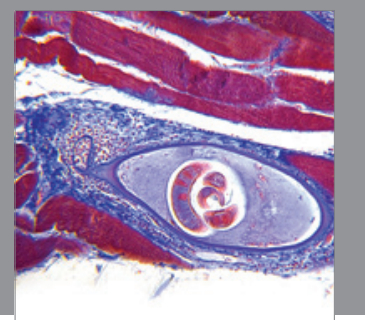

Gastroenterology

Research and Practice
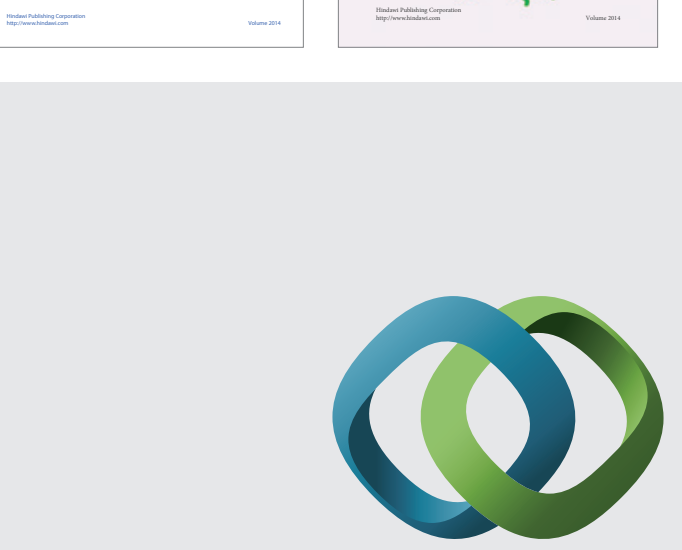

\section{Hindawi}

Submit your manuscripts at

http://www.hindawi.com
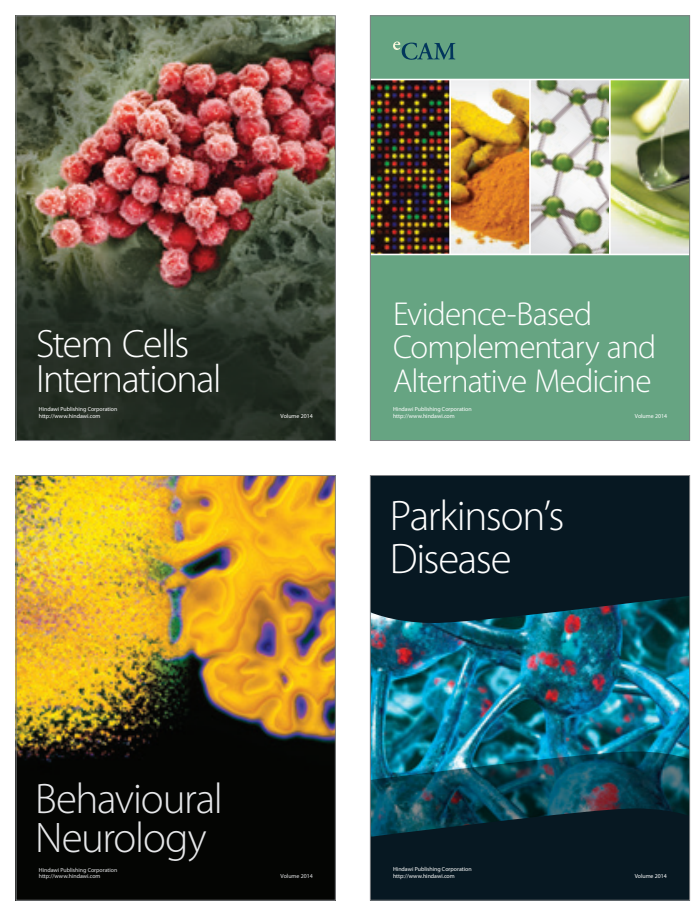

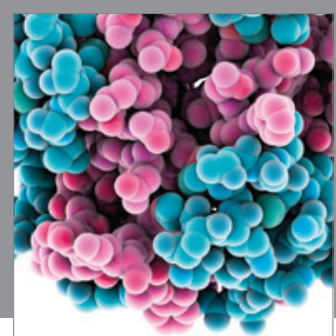

Journal of
Diabetes Research

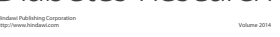

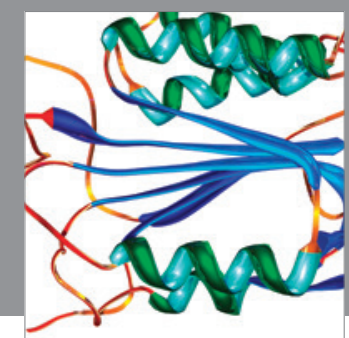

Disease Markers
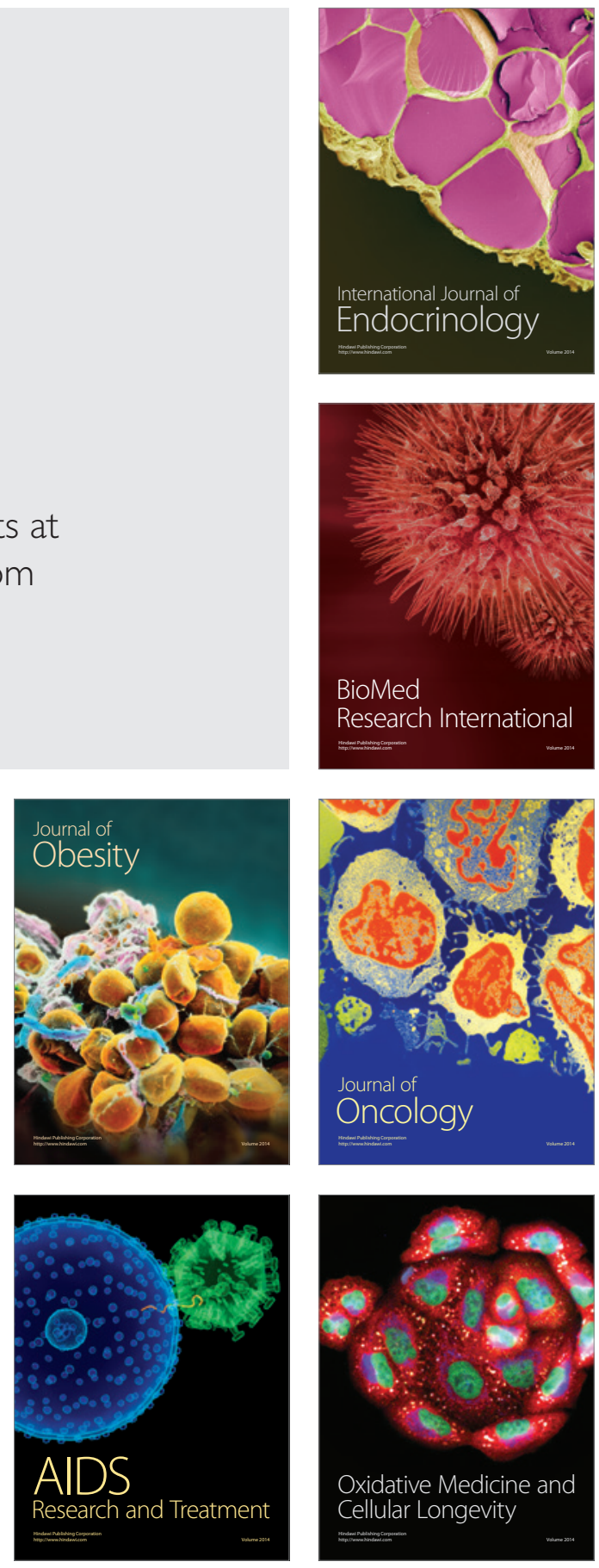